\title{
Que reste-t-il de la langue et de la culture grecques sur les côtes turques de la mer Noire?
}

Faruk Bilici

\section{OpenEdition}

1 Journals

Édition électronique

URL : https://journals.openedition.org/ceb/834

DOI : $10.4000 /$ ceb.834

ISSN : 2261-4184

Éditeur

INALCO

Édition imprimée

Date de publication : 30 mars 2011

Pagination : $325-342$

ISBN : 978-2-85831-189-7

ISSN : 0290-7402

Référence électronique

Faruk Bilici, «Que reste-t-il de la langue et de la culture grecques sur les côtes turques de la mer Noire? », Cahiers balkaniques [En ligne], 38-39 | 2011, document 18, mis en ligne le 17 février 2012 consulté le 06 juillet 2021. URL : http://journals.openedition.org/ceb/834 ; DOI : https://doi.org/ 10.4000/ceb.834

Ce document a été généré automatiquement le 6 juillet 2021.

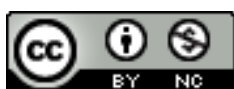

Cahiers balkaniques est mis à disposition selon les termes de la Licence Creative Commons Attribution - Pas d'Utilisation Commerciale 4.0 International. 


\title{
Que reste-t-il de la langue et de la culture grecques sur les côtes turques de la mer Noire?
}

\author{
Faruk Bilici
}

\section{NOTE DE L'ÉDITEUR}

Cet article est la version revue et actualisée d'une communication présentée au colloque international sur les Grecs d'Anatolie et d'Istanbul, 1821-1964, qui s'était tenu à l'Ecole française d'Athènes les 23-25 février 2006. Par ailleurs, une version turque et une version française, remaniées, ont paru récemment dans deux publications. La première : « Kardeniz'in Türkiye Kıyılarında Yunan Dili ve Kültürünün Kalıntıları », Cengiz Çağla, Haldun Gülalp (dir.),Semih Vaner Anısına,Avrupa Birliği, Demokrasi ve Laiklik, Istanbul, Metis, 2010 [A la memoire de Semih Vaner, L'Union européenne, la démocratie et la laicite], p. 57-70 ; la seconde : «Rumluk après rumluk : la survivance de la langue et de la culture grecques sur les côtes turques de la mer Noire », Anatoli, De l'Adriatique a la Caspienne, Territoires, Politique, Sociétés, Nouvelle série des Cahiers d'études sur la Méditerranée orientale et le monde turco-iranien (CEMOTI), dossier : Pour une nouvelle Ententebalkanique, Paris, éditions CNRS, 2010, pp. 233-251.

1 Parlée par quelques milliers de personnes, la langue pontique fait partie de ces nombreuses langues dites régionales en Turquie, qui ne sont pas écrites, encore moins enseignées, mais qui enrichissent le paysage culturel turc grâce aux interactions avec le turc, dans une période où les développements politiques intenses en direction des minorités, notamment kurdes, laissent présager un certain épanouissement dans l'avenir pour les langues en voie de disparition. Cet article est un premier essai sociohistorique sur une langue et une culture qui ne demandent qu'à renaître de leurs cendres aussi bien en Turquie qu'en Grèce. 
2 La convention entre la Grèce et la Turquie signée en janvier 1923 est le document qui légalise avec l'approbation des grandes puissances l'épuration ethnique et religieuse à grande échelle sur les deux rives de la mer Égée, épuration commencée au XIX ${ }^{e}$ siècle et renouvelée à plusieurs reprises. L'échange de population, sur le modèle de millet ottoman obligeait les 1500000 orthodoxes de Turquie à émigrer vers la Grèce, dont 322500 des côtes de la mer Noire, de Sivas et de Kastamonu, sans parler des 65-70 000 personnes tuées entre 1916-19231. Inversement 350000 musulmans sont renvoyés en Turquie, sans parler des centaines de milliers d'autres obligés de partir à cause du traité de Berlin de 1878. Comme on le sait, ce sont la minorité musulmane de la Thrace occidentale $(120000)$ et la minorité orthodoxe d'Istanbul $(120000)$ qui sont dispensées de cet échange. Lorsque le processus a été plus ou moins achevé en 1926, tout le monde pensait - et pense encore - que les racines linguistiques et humaines des vingt-huit siècles d'une présence hellénique avaient disparu par enchantement; que seules subsistaient les querelles concernant les communautés dites "minoritaires" définies par le traité de Lausanne. Ainsi, la citoyenneté turque n'étant pas encore - elle ne l'est toujours pas - le déterminant identitaire, les populations ponticophones musulmanes restant sur le territoire turc sont plongées dans une crise d'identité dans cette ambiance de nationalisme laïque jacobin. Tandis qu'en Grèce des centaines d'associations, de centres culturels et folkloriques sont créés, de nombreux travaux scientifiques sont menés afin de perpétuer le souvenir et le folklore (et non la culture ou la langue) pontique, en Turquie c'est le silence qui prévaut. On enveloppe la culture hellénique de la mer Noire d'un «lazisme » ironique tout juste pour pouvoir raconter des blagues belges; pire, de nombreux travaux sont publiés pour avancer que si des populations continuent à parler le pontique c'est qu'il s'agit de quelques personnes d'origine turque qui ont appris la langue des Rums, une fois en Grèce.

Partant de l'hypothèse que la vision unitaire de l'État-nation est sérieusement mise en cause par l'émergence de nouvelles élites, sous la pression internationale pour une meilleure intégration de l'Union européenne et un climat plus détendu entre les deux rives de la mer Égée, on peut aborder les problèmes relatifs aux minorités sans provoquer trop de réactions épidermiques aussi bien en Turquie qu'en Grèce. En attendant des recherches exhaustives, cette étude se limitera à aborder prioritairement trois questions : l'historiographie, la langue et la toponymie.

\section{Une histoire à construire}

4 Malgré les travaux éclairants de A. Bryer ${ }^{2}, \mathrm{H}$. Lowry ${ }^{3}$ et de M. Meeker ${ }^{4}$, l'histoire de la mer Noire sous l'angle de la langue et de la culture helléniques reste à construire. Dans la période ottomane, les lignes de démarcation entre religion et utilisation de la langue sont floues. H. Lowry surtout a bien montré que l'islamisation des populations pontiques n'a pas diminué l'utilisation de la culture et de la langue d'origine hellénique dans la région de Trabzon. Paradoxalement, les cartes sont encore plus brouillées dans la période contemporaine, car à la distinction religieuse se sont ajoutés le nationalisme et le déterminisme historique, la prétention de l'appropriation de la terre par une seule langue, une seule culture. Au grand dam des courants idéologiques et politiques nationalistes et fondamentalistes, ce qui nous intéresse ici c'est justement le phénomène de la cohabitation d'une langue et d'une culture considérées abusivement comme effacées de la géographie. 
5 Actuellement, deux tendances d'historiographie coexistent en Turquie quant à l'origine des populations et de la culture du Pont. La première, largement majoritaire et inaugurée par des historiens de la période de la «théorie langue-soleil » comme Osman Turan $^{5}$, Faruk Sümer ${ }^{6}$ et Fahrettin Kırzıoğlu ${ }^{7}$ émet des thèses très proches du pantouranisme. Selon cette historiographie, pratiquement tous les peuples ayant habité l'Asie mineure sont issus des Turcs de l'Asie centrale : notamment les Assyriens, les Hittites et les Cimmériens, les Scythes et bien d'autres. Elle n'hésite donc pas à trouver dans toutes les localités pontiques de profondes racines centre-asiatiques. Osman Turan (1914-1978) surtout en tant que ponticophone lui-même a joué un rôle fondamental. Historien médiéviste né à Çaykara (Kadahori) dans la région qui nous intéresse, Osman Turan a été député dans les rangs du parti démocrate (1954-1960) et il s'est retiré de la vie politique en 1969. Tout en poursuivant ses recherches et sa vie politique, il a surtout dirigé les « Foyers turcs » et a rédigé de nombreux articles dans la revue Turk Yurdu, les deux organes du pantouranisme. Les nombreux ouvrages sur l'époque Seldjoukide font d'Osman Turan une autorité sur l'histoire médiévale turque, mais ses prises de positions idéologiques et politiques se reflètent largement dans ses ouvrages qui fixent probablement encore pour longtemps le cadre de l'historiographie turque. Les thèses de cette historiographie concernant la turcité des pontiques sont bien résumées dans un ouvrage de micro-histoire de Haşim Albayrak, of ve Çaykara Tarihi (Histoire d'of et de Çaykara) ${ }^{8}$. Même s'il prend la précaution de dire qu'il ne peut pas l'affirmer avec certitude, Albayrak pense que, «les populations les plus anciennes de l'Anatolie, en conséquence celles de Trabzon, sont les Hittites, autrement dit les Turcs " ${ }^{9}$. Si Hérodote a dit que ces régions étaient colonisées par les Scythes, "c'est parce que le mot Scythe était utilisé pour désigner les Turcs Saka. Certains historiens appelaient également Saka, les Turcs qui vivait en dehors de l'Asie ${ }^{10}$. Selon Hasan Umur, un autre historien de la région d'Of, en 1019-1093, la région d'Of était le pays des Turcs kumans. Ils étaient installés entre Sinop et Batoum. Leur han s'appelait Ofşin (dans la langue turk kumane, Ofşin veut dire "défendre son pays avec acharnement, Of-ob, veut dire arme ») et leur capitale était of) ${ }^{11}$. Xénophon dans son Anabasis parle des peuples habitant à l'est de Trabzon, comme les Karduk, les Chilaps, les Scythes, les Maron-Koks, les Khaldis. Or ces tribus n'étaient rien d'autre que des Turcs $^{12}$.

6 Que faut-il déduire de tout cela ? Tout simplement qu'avant que les Grecs colonisent la région de Trabzon et d'of, des peuples habitaient ces contrées et que ces peuples étaient des Turcs. Or les Grecs qui fondèrent des colonies sur les côtes de la mer Noire hellénisèrent, puis christianisèrent ces Turcs, par leur civilisation, leur littérature.

7 Curieusement ces thèses reflètent le climat idéologique de la période de guerre des années de 1919-1923. En effet à la veille de la Conférence de Lausanne, tandis que les Grecs tentaient de prouver l'hellénisme de l'Asie mineure, les Turcs de leur côté rédigeaient des ouvrages pour «montrer comment l'Anatolie était turque depuis les origines de son histoire connue $»^{13}$. Encore plus curieusement, l'ouvrage qui avait servi de propagande contre les thèses de la Grèce, et publié en 1922 en turc et en français, avant même la conférence de Lausanne, est réédité en turc, en 1995, par la Grande Assemblée nationale. Son président Hüsamettin Cindoruk justifiait cette publication dans sa préface de la manière suivante :

8 «La réédition de cet ouvrage, dans la nouvelle langue, n'a pas de visées politiques. Nous n'avons pas comme objectif de nouveaux affrontements et débats. Nous souhaitons 
seulement que le monde scientifique renouvelle ses connaissances $»^{14}$. Or, dans cet ouvrage, un grand nombre de documents contemporains ont été publiés, et toute la partie introductive est consacrée à la turcité de l'Anatolie depuis toujours.

9 Ce sont ces thèses officielles qui sont reprises également par l'extrême droite turque pour dénier toute présence hellénistique linguistique et culturelle dans la région. Si on laisse de côté le problème ethnographique lié aux instruments de musique (kemençe, tulum) et aux tenues vestimentaires qui sont très influencés par les traditions caucasiennes, les tenants d'une région "purement turque » expliquent l'existence d'une langue grecque par un certain nombre d'arguments. D'abord le royaume pontique a été fondé en - 280 par la dynastie perse Mithridate qui n'a aucun lien avec les Grecs. Ce royaume a été annexé à l'Empire romain en - 63. Les Grecs sont venus dans cette région comme négociants et impérialistes; le Pont n'a jamais été donc grec, comme le prétend l'idéologie de Megali Idea. De toute façon, le mot rum n'a aucun lien avec le mot hellène ou grec. Car l'appellation rum, d'origine arabe est donnée à des populations touraniennes et perses. Par ailleurs, les Turcs se sont toujours approprié la langue de la religion qu'ils ont adoptée; étant donné que les Turcs anciens sont devenus chrétiens avec l'arrivée des Grecs, ils ont également adopté la langue. La preuve en est que les Bulgares d'origine turque sont devenus orthodoxes et ont adopté la langue slave. De même, si de grandes familles orthodoxes grecques portent des noms turcs comme Pehlivanoğulları, Oküzoğulları, Hırçınoğulları, şahinoğulları, Arslanoğlu, Kırbaşoğulları, Dumanoğulları, Karayamalı, c'est qu'elles étaient en réalité d'origine turque ${ }^{15}$.

10 Contre cet unanimisme, peu de voix discordantes s'élèvent. Le premier de ces travaux a paru en 1996 sous le titre de Pontus Kültürü̈ ${ }^{16}$.Son auteur, Ömer Asan est originaire d'un village de l'arrondissement d'of, Çoruh, village ponticophone. L'ouvrage est autobiographique puisqu'il s'agit d'un témoignage. L'auteur a compilé un grand nombre de données relatives à la langue, à la culture, à la toponymie d'of et plus particulièrement de son village à travers sa famille et son entourage. La crainte d'être déconsidéré et de passer pour un « traître » est lisible dans son introduction :

11 «Il y aura des gens qui chercheront des arrière-pensées dans cette étude. Je sais que les milieux obsédés depuis toujours par certaines idées fixes considéreront ce livre comme une œuvre 'visant à diviser la Turquie', je leur conseille de jeter un coup d'œil non pas à toute l'histoire, mais seulement de la lire sur cent ans. Ils verront que les hommes de différentes religions, de langues et de cultures vivaient dans la paix. Mais malgré cela, je sais qu'ils n'en feront qu'à leur tête. Cette vision qui cherche le bonheur de la Turquie dans les méthodes d'épuration ethnique ou dans la suppression physique est triste, et elle est toujours en vigueur $»^{17}$.

12 En effet, cette prophétie s'est réalisée en grande partie. Car, réédité en 2000, l'ouvrage d'Asan a fait, deux ans après, dans la frénésie d'une campagne médiatique pour "sauver la Turquie des griffes d'une christianisation par les missionnaires », l'objet d'une dénonciation virulente dans la presse islamiste et nationaliste. Mais, après la saisie de son livre, il est surtout déféré devant le tribunal de sûreté d'Istanbul, sous le prétexte de "propagande écrite dans le dessein de nuire à l'unité de l'État et de la nation de la République turque ». La justice militaire reprochait à l'auteur comme à son éditeur, Ayşe Zarakolu d'avoir fait rédiger la préface à un universitaire grec, Neoklis Sarris, où celui-ci disait notamment que ce sont les mêmes raisons officielles qui ont présidé, après la Première Guerre mondiale, au débarquement de Mustafa Kemal à 
Samsun, comme à celui de la Grèce en Anatolie. Selon Sarris, accusé d'ailleurs par les plaignants d'aider la branche du PKK, installée dans son pays, dans les deux cas, ce sont des débarquements qui ont été approuvés et encouragés par les Alliés pour protéger la communauté grecque des bandes armées musulmanes ${ }^{18}$. Par ces propos et bien d'autres activités qui lui sont attribués, $\mathrm{N}$. Sarkis chercherait à séparer la région de la mer Noire pour en faire un État pontique. De la même manière, Ö. Asan, était accusé d'avoir affirmé, surtout par la présentation d'un dictionnaire turc-rum du dialecte utilisé actuellement, que des populations influencées par la culture pontique vivent toujours sur les côtes de la mer Noire et surtout dans la partie orientale, à Trabzon, à of et à Çoruk (Erenköy) ${ }^{19}$. L'auteur a eu l'occasion de démontrer l'absurdité des accusations, mais, c'est à la faveur des réformes adoptées par le gouvernement en place pour se conformer à la législation européenne en supprimant l'article 8 de la loi anti-terroriste que l'auteur a été relaxé et son ouvrage remis en circulation ${ }^{20}$.

La seconde publication vient de voir le jour. Il s'agit d'un dictionnaire encyclopédique de la mer Noire (Karadeniz Ansiklopedik Sözlük), en deux volumes, rédigé par un jeune chercheur Özhan Öztürk ${ }^{21}$, qui, visiblement, a tout fait pour ne pas attirer l'attention des autorités judiciaires. Mais cela ne l'a pas empêché d'être désigné comme un auteur manipulé par Athènes. Publié aux éditions Hayamola, créées par Ö. Asan, ce dictionnaire est le premier du genre à intégrer autant de concepts, de mots, de noms grecs dans un ouvrage. Réalisé à la suite d'une enquête longue de collecte dans les départements d'Ordu, Giresun, Trabzon, Rize et Artvin, le dictionnaire met en évidence les mots et noms, entre autres, d'origine grecque, mais surtout il retrace brièvement l'histoire de chaque localité. Le folklore est largement utilisé aussi bien sous forme de chansons que de poèmes ou d'expressions. En tant qu'intellectuel local «ayant des devoirs envers ses aînés, Özhan Öztürk, loin des préoccupations idéologiques ", se fixe pour objectif de collecter, d'interpréter le patrimoine culturel et de le transmettre sans interruption aux générations futures ${ }^{22}$.

point de départ de ces deux ouvrages est identique : les auteurs, non professionnels de la recherche, se sont posé la question de savoir pourquoi leur entourage, les gens de leurs villages et eux-mêmes parlaient une langue différente du turc, de surcroît une langue dont les locuteurs principaux ont été déportés. Nous touchons ainsi à la question de la définition de l'identité pontique qui se construit avant tout par un territoire particulier et une langue spécifique ${ }^{23}$. À ces deux éléments, il faut ajouter certaines pratiques typiques comme la cuisine, le folklore, les soins du corps, des pratiques sociales comme la parenté, les structures politiques, la tradition orale.

\section{Une langue en voie de disparition}

15 La dévalorisation et le dénigrement systématiques de l'histoire sont également valables pour les travaux mettant en avant la langue et la culture grecques dans la région de Trabzon.

Il a fallu attendre le recensement de 1965 où l'on a posé la question de savoir quelle était la langue maternelle de chacun, pour « découvrir » que la langue et la culture grecques n'avaient pas disparu dans la région de Trabzon. Les résultats de ce recensement ont été exploités par Peter Alford Andrews et une équipe de chercheurs, et édités seulement en 1989, sous le titre d'Ethnic Groups in the Republic of Turkey ${ }^{24}$. La seconde édition de cet ouvrage est faite en 2002, mais elle n'a rien ajouté en ce qui 
concerne les populations musulmanes parlant le grec pontique. Les Pontiques se désignent comme turcs ou encore Oflis.

Tableau I : Habitants des localités parlant le grec pontique (recensement de 1965) ${ }^{25}$

\begin{tabular}{|c|c|c|}
\hline Département / commune & Arrondissement & Canton \\
\hline SAKARYA & Hendek & Karadere \\
\hline Dikmen & « & « \\
\hline Göksu & « & « \\
\hline TRABZON & Çaykara & Çaykara \\
\hline Akdoğan (Yukarı Hopşera) & « & « \\
\hline Aşağıkumlu (Aşağı Mimiloz) & « & « \\
\hline Blatacılı & « & « \\
\hline Çambaşı (Anoso) & « & « \\
\hline Eğridere (Gorgoras) & « & « \\
\hline Kabataş (Fotinos) & « & « \\
\hline Karaçam (Yukarı Ogena) & « & « \\
\hline Kayran (Limli) & « & « \\
\hline Koldere (Vahtanç/Paçanvahtanç) & « & $\ll$ \\
\hline Köknar (Aşağı Ogena) & « & « \\
\hline Maraslı (Nefsipaçan) & « & « \\
\hline Soğanlı (Asağı Hopşera) & « & « \\
\hline şahinkaya (şur) & « & « \\
\hline Taşligedik (Mezireipaçan) & « & « \\
\hline Taşeren (Zeleka) & « & $\ll$ \\
\hline Uzuntarla (Alisinos) & « & « \\
\hline Yeşilalan (Holaysa) & « & « \\
\hline Yukarı Kumlu (Yukarı Mimiloz) & « & « \\
\hline TRABZON & Çaykara & Dernekpazarı \\
\hline
\end{tabular}




\begin{tabular}{|c|c|c|}
\hline Dernekpazarı (Kondu) & « & « \\
\hline Çalışanlar (Kalanas) & « & « \\
\hline Çayırbaşı (Fotrekan) & « & « \\
\hline Günebakan (Zenozeno) & « & « \\
\hline Ormancik (Mekidanoz/Holomaktanoz) & « & « \\
\hline Taşçılar (Fotgene) & « & « \\
\hline Tüfekçi (Arşala) & « & " \\
\hline Yenice (Zaimler) & « & « \\
\hline TRABZON & Çaykara & Uzungöl \\
\hline Uzungöl (şerah/Saraço) & « & « \\
\hline Demirli (Kotlu) & « & « \\
\hline Derindere (Asafoliza) & « & « \\
\hline Köseli & « & « \\
\hline Şekersu (Sakarsu) & « & « \\
\hline Taşkıran (Coroş) & « & « \\
\hline TRABZON & Maçka & Maçka \\
\hline TRABZON & of & Hayrat \\
\hline Yeniköy & « & « \\
\hline TRABZON & Sürmene & Sürmene \\
\hline Dirlik (Zide) & « & « \\
\hline TRABZON & Sürmene & Köprübaşı \\
\hline Büyük Doğanlı (Büyük Arhançılo) & « & « \\
\hline Dağardı (Okasho) & « & « \\
\hline Konuklu (Halis/Halith) & « & « \\
\hline Küçük Doğanlı (Küçük Aharançılo) & « & « \\
\hline Yilmazlar (Holomezrea) & « & « \\
\hline
\end{tabular}




\begin{tabular}{|c|c|c|}
\hline TRABZON & Tonya & Tonya \\
\hline Tonya meziresi, Büyükmahalle & « & « \\
\hline İskenderli (Kumyatak, Skandarando) & $«$ & $\ll$ \\
\hline Kozluca & $\ll$ & « \\
\hline Melikşah (Melikşe) & « & « \\
\hline Sayraç & « & « \\
\hline Turalı & « & « \\
\hline Yakçukur & $\ll$ & « \\
\hline TRABZON & Vakfikebir & Beşikdüzü \\
\hline Dağlıca (Mesopliya) & « & « \\
\hline
\end{tabular}

Selon ce recensement, 4535 personnes ont déclaré avoir comme langue maternelle le grec dans la région de Trabzon, et 900 autres personnes dans d'autres provinces. Mais nous nous intéresserons aux premières qui se situent essentiellement dans les arrondissements de Çaykara, Of, Sürmene, Tonya et Maçka (Matzouka), réparties sur 53 localités (villes, bourgs et villages).

Il est évident que le chiffre révélé par le recensement paraît bien dérisoire par rapport à la population qui parlait réellement le grec à cette époque, rien que parce que certains villages parlant aujourd'hui le pontique n'y sont pas. L'exemple le plus frappant est celui de Çoruh, ce village dépendant d'of et à partir duquel Ö. Asan a écrit l'ouvrage dont il a été question plus haut. Par ailleurs, le nombre de personnes qui ont accepté d'emblée que leur langue maternelle était le grec est totalement sujet à caution. Ceci était problématique à l'époque et l'est toujours. La dévalorisation des langues et des cultures régionales dans les États-nations issus de l'Empire ottoman est une constante. Surtout dans le cas d'une population parlant une langue cataloguée comme celle d'une minorité non-islamique. Il est plus facile par exemple d'accepter comme langue maternelle le tcherkess, l'abkhaz ou même le kurde que le grec ou l'arménien, identifiés clairement comme des langues de "gavours" ayant posé problème à la fin de l'Empire ottoman. En effet, rares sont les locuteurs pontiques qui acceptent ouvertement d'être les descendants des Rums, alors que des personnes âgées qui ne parlent pratiquement pas le turc sont encore nombreuses ${ }^{26}$. Selon les estimations, sur une population de 975000 personnes sur l'ensemble du département de Trabzon, 300000 (33\%) parlent le pontique. Il faut dire que ces estimations ne sont basées sur aucune étude scientifique. La meilleure manière d'avoir une certitude c'est d'entreprendre des enquêtes linguistiques, ethnologiques, anthropologiques et sociologiques au moins dans les localités réputées grécophones. Mais la tâche paraît difficile, tant il est vrai que les autorités y verraient une tentative de "séparatisme ", alors qu'au-delà de toute polémique, il s'agirait de saisir, de décrire et d'enregistrer une 
langue, une culture et un folklore exceptionnels dont l'empreinte s'efface de jour en jour.

19 La langue pontique constitue un ensemble dialectal relativement homogène, surtout du point de vue morphosyntaxique. Le dialecte de of-Sürmene présente quelques particularités remarquables qui ont été décrites plusieurs fois, notamment par Peter Mackridge dans une courte étude ${ }^{27}$, puis récemment dans une thèse magistrale par Georges Drettas ${ }^{28}$. Selon ce dernier, l'obsolescence de cette langue est programmée ${ }^{29}$; elle est sur le point de mourir, puis qu'elle n'est enseignée ni en Turquie ni en Grèce ; les partisans de sa disparition sont légion des deux côtés ; peu de locuteurs la parlent: en Grèce, la réalité culturelle des populations exogènes a été systématiquement marginalisée et donc du coup les dialectes y sont condamnés à disparaître. Dans tous les cas, les partisans de l'obsolescence assimilent la ponticophonie à une alloglossie ${ }^{30}$. Tandis qu'en Turquie, c'est l'indifférence, l'uniformité linguistique, culturelle et religieuse, voire les contraintes qui sont en train de la tuer. C'est donc dans les familles et surtout dans l'espace intime qu'elle est utilisée comme langue quotidienne et uniquement orale, sans jamais passer par l'écrit. Dans la dévalorisation des cultures régionales, elle a été considérée comme une tare honteuse, confinée dans le folklore le plus réducteur.

20 Nous donnerons deux exemples de la langue pontique telle qu'elle est parlée dans deux villages de Çaykara (Khadahori) : Yukarı Ogena (Karaçam) ${ }^{31}$ et Alisinos (Uzuntarla) ${ }^{32}$. Naturellement dans ce genre de texte oral l'alphabet grec n'étant pas utilisé, la transcription en $\operatorname{grec}^{33}$, puis en lettres latines reste problématique et forcement approximative.

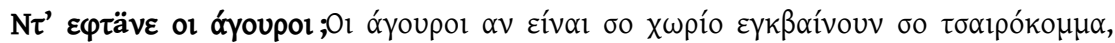

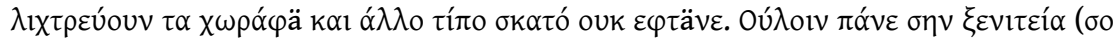

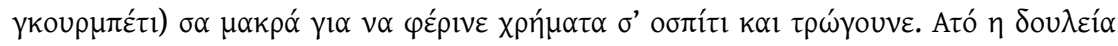

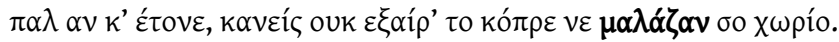

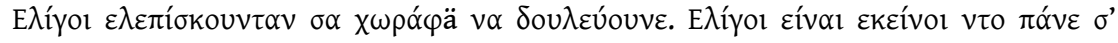

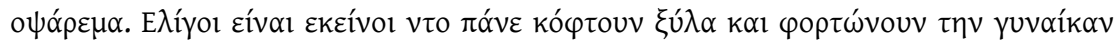

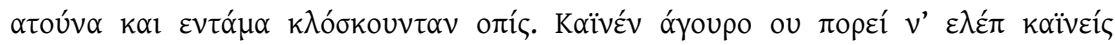

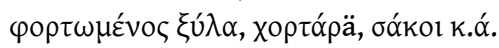

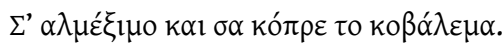

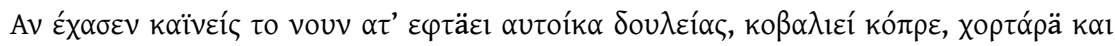

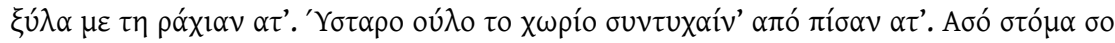

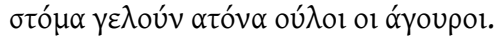

Nt'eftene $i$ aguri?

I aguri an ine so xorio egvenun so ceyrokoma, lixtrevun ta xorafe ce alo tipo skato uc eftene. Ulin pane sin ksenitia (so gurbeti) sa makra ya na ferine xrimata s'ospiti ce trogune. Ato i dulia pal an c'etone kaynis uc ekser to kopre ne malazan so xorio.

Eligi elepiskuntan sa xorafe na dulevune. Eligi ine ecini nto pane s'opsarema. Eligi ine ecini nto pane koftun ksila ce fortonun tin yinekanatuna ce entama kloskuntane opis. Kaynen aguro u'poriy nelep kaynis fortomenos ksila, xortare, saci c.a.

S'almeksimo ce sa kopre to kovalema...

An exasen kaynis to nunat eftey aytika dulias, kovaliy kopre, xortare ce ksila me ti rashanat. Istaro ulo to xorio sintishen apo pisanat. Aso stoma so stoma yelunatona uli i aguri.

Que font les hommes?

Lorsque les hommes sont au village, ils vont faucher de l'herbe, bêcher le champ et ne foutent rien d'autre. En général, pour ramener de l'argent à la maison et le dépenser, ils partent tous loin. Et si ce boulot n'existait pas on se demande ce qu'ils pourraient bien foutre. 
Quelques-uns vont travailler dans les champs. Peu parmi eux vont à la pêche. Peu nombreux sont ceux qui vont couper du bois, charger leurs femmes et rentrer à la maison avec elles. Personne ne voit les hommes chargés d'herbes, de sacs etc.

On ne les voit pas, non plus traire les vaches et porter du fumier.

Si quelqu'un perd la tête, alors il peut bien faire ces choses-là : porter sur le dos du fumier, de l'herbe et du bois. Par la suite tout le village dit du mal de lui derrière son dos. La nouvelle va de bouche en bouche et il devient la risée de tous les hommes.

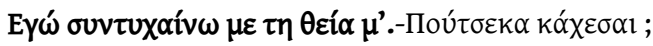

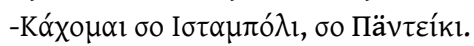

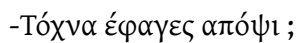

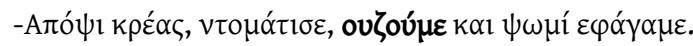

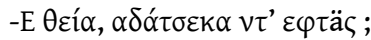

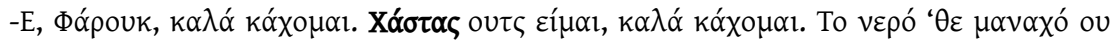
$\pi \circ \rho \omega ́ v \propto \alpha i ́ v \omega$.

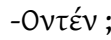

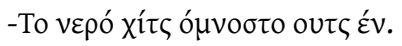

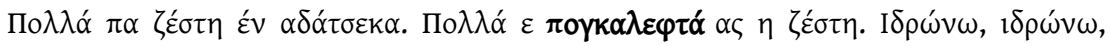

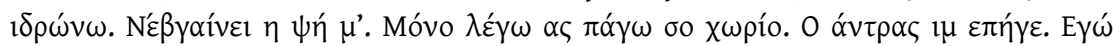

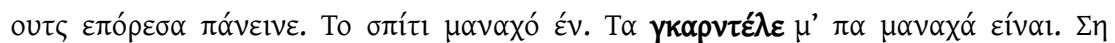

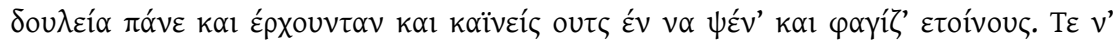
$\varepsilon \varphi \tau{ }^{\prime} \gamma \omega$;

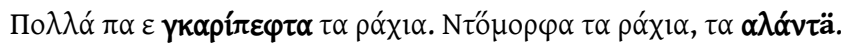

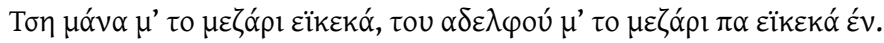

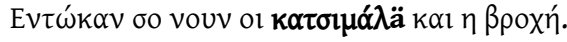

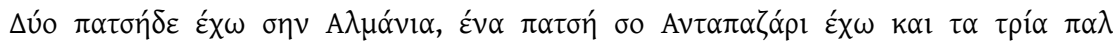

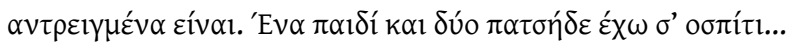

Egosintishenometithiyam.

- Puçekakahese?

- KahomesoIstanboli, soPendiki.

- Tohnaefayesapopsi?

- Apopsikreyas, domatise, uzumecepsomiefagame.

- Ethiya, adaçekadeftes?

- EFarukKalakahome. Hastas uç ime, kala kahome. To nerothe manaho u poro na pino.

- Oden?

- To nero hiç omnosto ucen.

Pola pa zesti en adaceka. Pola e pogalefta asi zesti. Idrono, idrono, idrono. N'egveni i pshim... Mono lego as pago so horiyo. 0 Antrasim epiye. Ego uç eporesa panine. To spiti manaho en. Ta gardelem pa manaha ine. Si duliya pane ce erhuntan ce kaynis ucen na psen ce fayizetinus. Te n' eftago?

Pola pa e garipefta ta rashiya. Nt' omorfa ta rashiya, ta alade...

Tsi manam to mezari eyçeka, tu adelfum to mezari pa eyçeka en.

Entokan so num i katsimale ce $i$ vroshi

Diyo patside eho sin Almanya, ena patsi so Adapazari eho ce ta triya pal antrigmena ine. Ena pedi ce diyo patside eho s'ospiti...

Je m'entretiens avec ma tante.

Où habites-tu?

J'habite à Istanbul, à Pendik.

Qu'as-tu mangé ce soir?

Ce soir, nous avons mangé de la viande, des tomates, du raisin et du pain.

Ma tante, que fais-tu ici?

Ô Faruk, je suis bien installée. Je ne suis pas malade. Seulement, je ne peux pas boire de l'eau [d'ici].

Pourquoi?

Elle n'a aucun goût. Ici.

Il fait trop chaud. La chaleur me dérange beaucoup. Je transpire, transpire, 
transpire. J'en meurs.

Parfois je me dis qu'il faut que je parte dans le pays [à Trabzon]. Mon mari est parti. Je n'ai pas pu partir. La maison est toute seule. Mes enfants sont seuls. Ils vont au travail, ils reviennent à la maison ; personne ne leur fait à manger. Que faire ?

J'ai beaucoup de nostalgie des montagnes, des belles montagnes, des pins !

La tombe de ma mère est là-bas ; celle de ma sœur est là-bas aussi.

Lorsque le brouillard arrive, il y pleut.

J'ai deux filles en Allemagne, une autre à Adapazarı; j'ai donc trois filles mariées ; j'ai un fils et deux filles à la maison...

cela dit, des différences existent entre les régions et même entre les villages. L'exemple le plus frappant est celui qui est signalé par P. Mackridge. Il s'agit des noms de mois à Saraho et dans le reste du Pont.

Tableau : II Les noms des mois en Ophitic ${ }^{34}$

\begin{tabular}{|l|l|}
\hline Saraho & En général sur le Pont \\
\hline o kalandaris & o kala-azars \\
\hline o kunduron & o kunduron \\
\hline o martis $\left(^{*}\right)$ & o marts \\
\hline o aprilis $\left(^{*}\right)$ & o aprilits \\
\hline o mais $\left(^{*}\right)$ & o karyokinèses \\
\hline o kirezis & o kerasinon \\
\hline o thurukis & o kolatiers/ferinon \\
\hline o aguston $\left(^{*}\right)$ & o aguston / avguston \\
\hline o stavrites & o stavrites \\
\hline o xtombris $\left(^{*}\right)$ & o triominas \\
\hline o triyominon & o aerjites \\
\hline o xtsjonaris / ksjonaris & o xristjenaris \\
\hline
\end{tabular}

$\left.{ }^{\star}\right)$ : les noms utilisés en grec moderne.

21 Le grec pontique est influencé considérablement par le turc, bien sûr sur le plan lexicologique, mais aussi sur le plan syntaxique. Par exemple, sur 409 verbes recensés (parfois avec la forme transitive) ${ }^{35}, 25$ (6,11\%) sont d'origine turque, mais ils sont conjugués en pontique, tels que ankevo (se rappeler, appeler), arayevo (je cherche); yerleşevo (je m'installe), yakalayevo (j'attrape), yanlayevo (j'esquive), yarayevo (je suis utile), yitirevo (je perds) ou encore zorlayevo (je force), inanevo (je crois), kanture va (je trompe), keçinevo (je survie), güvenego (j'ai confiance), kourevo (je monte je construis), koutourevo (je rage), ugrachevo (je m’occupe), pegenefgume (nous nous apprécions), puchmanevo (je renonce) etc. 
région. Dans un dictionnaire étymologique établi à partir du turc parlé dans l'arrondissement de Maçka (Maztouka), Trabzon-Maçka Etimoloji Sözlüğü (Dictionnaire étymologique de Trabzon-Maçka) ${ }^{36}$, l'on constate que, non seulement le principe fondamental, l'harmonie vocalique, a disparu de la langue, mais un grand nombre de mots d'origine pontique ont fait leur entrée dans la langue quotidienne. Ainsi selon le dictionnaire de Kudret Emiroğlu, sur 2145 mots recensés 727 (33,9\%) sont en turc, 549 $(25,6 \%)$ d'origine inconnue, $360(16,5 \%)$ en grec, $206(9,6 \%)$ en arabe, $150(7 \%)$ en persan, le reste respectivement en italien, russe, arménien et autres. Or si le dictionnaire était bien fait on n'aurait pas laissé tant de mots d'origine inconnue et parmi ces derniers, une partie serait recensée comme étant d'origine pontique.

Il n'existe pas encore d'étude systématique des toponymes des anciennes provinces pontiques. En ce qui concerne les noms de villages ou de bourgs, nous n'avons pas de problème particulier, car ils n'avaient pas été modifiés à l'époque ottomane. Durant les années 50 et $60 \mathrm{du} \mathrm{XX}$ siècle, la politique de changement de noms sur les côtes de la mer Noire s'est brusquement emballée pour remplacer systématiquement tous les noms grecs de la région de Trabzon par des noms turcs, très souvent sans aucun rapport avec l'origine du nom. Ainsi en 1973, sur 562 villes et villages 431 (77\%) portaient encore des noms anciens essentiellement grecs ${ }^{37}$. Dans certains arrondissements comme Çaykara, of ou encore Maçka, pratiquement tous les villages avaient un nom grec, tandis que dans d'autres, notamment à l'ouest, ce pourcentage descend par exemple à Beşikdüzü à $32 \%$, à Vakfikebir à $20 \%$, ou encore à Çarşıbaşı (Iskefiye) à $14 \%$. Ces changements semblent s'être faits de manière arbitraire.

En revanche, les noms de lieux-dits, tels qu'ils existent aujourd'hui, peuvent être révélateurs pour mesurer la vivacité de la culture pontique dans l'arrondissement d'of. Aussi pour le village de Çoruk par exemple, Ö. Asan a énuméré soixante-six noms de lieux-dits dont cinquante-quatre ( $78 \%$ ) sont pontiques, comme Stali, Kufald, Vizlando, Ahontona, Sintoma, Kosara kabani ou encore Holaho Mantora etc. ${ }^{38}$. De notre côté, nous avons tenté de repérer dans un autre village, à Alisinos (Uzuntarla), les noms des lieuxdits et quartiers existants : Divren, Suyamando, Ahmadan, Potami, Hobchera d'Ormi, Dafia, Ragan, Nklisiya, Barhari, Balgodi, Aplayeras, Avlago Thcofala, Mavreyas, Lichia.

\section{Conclusion}

Ce travail sur la toponymie peut se faire sur les noms de famille, devenus les surnoms ou les noms de réputation depuis que la législation turque a imposé à chacun de choisir un nom de famille. En effet, la loi du 21 juin 1934, impose des noms turcs et elle interdit d'utiliser des noms "étrangers». Du coup, toute une gamme de noms de familles pontiques a disparu officiellement dans la région de Trabzon, mais elle subsiste dans la vie quotidienne permettant leur identification. Ainsi à Çoruk, nous avons, à côté d'un certain nombre de noms turcs (Ahmetaliler, Hacaliler, Eminliler, Memişliler, Koloğulları, Kısıroğulları, Karahacılar, Ömerustalar, Küçükoğulları, Bahadırlar, Imamlılar, etc.), plusieurs noms de famille grecque: Skolekallar, Vintalılar, Hentemliler, Maritsalılar, Tsileyalılar, Kosaralılar etc. Autrement dit sur 78 noms de familles comptés, 33 ( $42 \%)$ sont identifiés comme d'origine turque, les autres sont essentiellement d'origine grecque. Ce n'est pas aussi exhaustif, mais le petit recensement que nous avons réalisé dans deux petits villages, Alisinos et Ogena, donne 
également des surnoms de familles comme Gogoç, Tsileya, Galegoglu, Yanukh, Gucala, Danomalezo, Nefter, Kakoç, Durko, Bebeşan Zumbi, Silil, Suls, Komara, Memen, Çahuc, Kazoradi, Rogra, Kinca, Kulukhtera, Salimadi, Ormiya, Begav, etc. Dans la petite ville de Skandarando, près de Beşikdüzü, de nombreux noms de familles grecs ont été repérés : Pakaloslar, Xulukolara, Momantlar, Xeskaloslar... sont seulement certains d'entre eux ${ }^{39}$. Dans tous les cas, un dictionnaire onomastique de la région de la mer Noire reste à faire.

La survivance de la langue, l'existence de cette toponymie, de l'onomastique, ainsi que d'un grand nombre de pratiques religieuses issues des traditions chrétiennes orthodoxes ont induit en erreur certains chercheurs et universitaires grecs qui s'intéressent à la question pontique. Probablement faute de travail sur le terrain ils n'hésitent pas à construire un monde imaginaire autour du concept chrypto-chrétien. Ils ont du mal à comprendre, comme les nationalistes et fondamentalistes turcs d'ailleurs, que l'on puisse avoir des identités multiples ; qu'une population puisse être musulmane tout en parlant le grec, et classent tout simplement les grécophones de la mer Noire dans la catégorie de " chrypto-chrétiens " pour expliquer cette " anomalie ». Ils préfèrent utiliser ce résidu de la langue et de culture comme une arme de propagande plutôt que comme un simple fait historique, sociologique et ethnologique méritant une attention particulière.

Pour mieux démontrer l'absurdité des thèses relatives au chrypto-christianisme, deux éléments doivent pouvoir être pris en compte. Le premier, c'est le phénomène de formation du clergé musulman, dans la période entre 1930 et 1950 où l'enseignement religieux était interdit. En effet, les arrondissements les plus profondément pondicophones, comme of et Çaykara étaient également ceux qui ont fourni le plus de clergé (imams, hâfiz, mufti) à toute la Turquie.

L'autre élément est l'analyse du paysage politique actuel dans la région qui nous intéresse. Les résultats électoraux dans le département de Trabzon en 2002 étaient assez différents par rapport aux moyennes nationales ${ }^{40}$. Les deux partis islamistes, le parti de la justice et du développement (Adalet ve Kalkınma Partisi, AKP) et le parti de bonheur (Saadet Partisi, SP) ont réuni dans le département, à eux deux, 49,5\% des voix : $43,9 \%$ (moyenne nationale : 34,2 ) et 5, $6 \%$ (m.n : 2,4 \%), alors que les autres partis de droite comme le parti de la Juste voie (DYP) et le parti du mouvement national (Milliyetçi Hareket Partisi, MHP), un parti nationaliste, obtenaient respectivement 10,7 \% (m.n: 9,5) et 10,7\% (m.n: 8,3). Le reste des voix sont allées aux autres partis : le parti républicain du peuple (Cumhuriyet Halk Partisi, CHP) : 14,6 \% (m.n : 19,4 \%) ; Jeune parti Genç Parti, GP) : 1,6\% (m.n: 7,2); le parti populaire démocratique (Demokratik Halk Partisi, DEHAP) : $0 \%$ (m.n: 6,2); le parti de la mer Patrie (Anavatan Partisi, ANAP) : 8 (m.n : 5,1) ; le parti démocratique de gauche (Demokratik Sol Parti, DSP ) : 1,3\%.

Aux élections législatives du 27 juillet 2007, cet écart est encore plus important, car le parti de la justice et du développement au pouvoir obtient tout seul la majorité absolue avec 231292 voix (56,8\%) sur un total de 407461 voix valables dans le département de Trabzon. Ainsi l'AKP rafle du même coup les sept sièges de députés sur huit attribués au département, le huitième revenant au principal parti d'opposition, le CHP. Sinon, le parti du mouvement national a obtenu 57294 voix (14\%) le parti républicain du peuple 55616 voix (13,6\%), le parti démocrate 25410 (6,2\%) voix, le reste des voix étant partagé par une multitude de partis. 
La présence de la langue et de la culture pontiques en Turquie est l'une des richesses du pays. Leur place est encore loin d'être appréciée à sa juste valeur aussi bien par les chercheurs que par les élites politiques. C'est seulement lorsque des recherches pluridisciplinaires seront entreprises que le potentiel historique, linguistique et sociologique de cet héritage culturel sera reconnu.

\section{NOTES}

1. Stefanos Yerasimos, Milliyetler ve Sinırlar, Iletişim Yayınları, Istanbul, 1994, p. 424.

2. Quelques ouvrages d'Anthony Bryer : The Empire of Trebizond and the Pontos, Variorum reprints, Londres, 1980, 366 p. ; avec David Winfield, The Byzantine monuments and topography of the Pontos, 2 vols, Dumbarton Oaks Research Library and Collection, Washington D.C, 1985 ; Peoples and settlement in Anatolia and the Caucasus, 800-1900, Variorum reprints, Londres, 1988.

3. Son ouvrage principal sur Trabzon: The Ottoman Tahrir Defters as a source for urban demographic history: The case study of Trabzon (ca. 1486-1583), Los Angeles, 1977.

4. Parmi les nombreuses études de M. Meeker, il faut surtout signaler ces deux ouvrages: Social practice and political culture in the Turkish Republic, Isis, Istanbul, 2004, 368 p.; A nation of Empire : the Ottoman legacy of Turkish modernity, University of California Press, Berkeley, 2002, XXVIII+420 p.

5. Voici trois titres seulement parmi l'œuvre considérable d'Osman Turan : Selçuklular Tarihi ve Türk-Islam Medeniyeti (1965), Université d'Ankara, Ankara, 1965, XIII-448 p. ; Selçuklular Zamanında Türkiye, Turan Neşriyat, Istanbul, XXX+752 p.; Doğu Anadolu Türk Devletleri Tarihi, Istanbul Matbaası, Istanbul, 1973, XVI+281 p.

6. Faruk Sümer (1924-1995) est connu surtout par ses ouvrages sur les Oguz, ancêtres des Turcs mais aussi par ses relations avec des organismes nationalistes, comme avec le gouvernement et surtout avec le ministère de l'Éducation nationale (il a rédigé plusieurs manuels d'histoire pour les établissements scolaires) : Oguzlar (Türkmenler) : Tarihleri, Boy Teşkilatı, Destanları (5 édition), Türk Dünyası Araştırmaları Vakfı, Istanbul, 1999, 416 p. ; Selçuklular Devrinde Doğu Anadolu'da Türk Beylikleri, Türk Tarih Kurumu Yayınları, Ankara, 1998; Kara Koyunlar, Türk Tarih Kurumu Yayınları; Ankara, 1990 ; Safevi Devleti'nin Kuruluşu ve Gelişmesinde Anadolu Türklerinin Rolü, Türk Tarih Kurumu Yayınları, Ankara, 1999.

7. Mehmet Fahrettin Kırzıoğlu (1917-2005) est un historien très célèbre qui s'est ingénié à "prouver» dans des ouvrages que les Kurdes étaient " des parfaits turcs »: Kürtler'in Kökü (Première partie), Ayyıldız Matbaası, Ankara, 1963 ; Her Bakımdan Türk Olan Kürtler, Ankara, 1964, 123 p.; Kürtler'in Türklüğ̈̈, Kurtuluş Matbaası, Ankara, 1968, 134 p. ; Dağıstan-Aras-Dicle-Altay ve Türkistan Türk Boylarından Kürtler, Ankara, 1984.

Membres de plusieurs organismes nationalistes, Kırzıoğlu a rédigé de nombreux ouvrages sur l'histoire de Kars, sur les Arméniens et les Turcs anciens.

8. Haşim Albayrak, Of ve Çaykara Tarihi, Cantekin Matbaası, Ankara, 1986.

9. Id. vol. 1, p. 104 .

10. Id. vol. 1, p. 105.

11. Id. vol. 1, p. 105.

12. Id. vol. 1, p. 106.

13. Yilmaz Kurt, Pontus Meselesi, Türkiye Büyük Millet Meclisi, Kültür, Sanat ve Yayın Kurulu Yayınları, numéro, 68, Ankara, 1995, p. 3-12. 
14. Id., préface.

15. http///www.turan.tc/pondusdosyasi

16. Istanbul, Belge Yayınları, 1996.

17. Ömer Asan, Pontus Kültürü, p. XXIV.

18. Ö. Asan, op. cit., p. XVII.

19. Acte d'accusation du procureur général de la République, devant la sixième chambre de sûreté d'Istanbul.

20. Décision de la sixième chambre de sûreté d'Istanbul, le 12 août 2003 . Voir aussi le site internet : http://www.omerasan.com.

21. Publié chez Hayamola, Istanbul, 2005, 1262 p.

22. Özhan Öztürk, Karadeniz Ansiklopedik Sözlük, introduction, p. IV.

23. G. Drettas, "La langue pontique comme objet identitaire : questions de représentations », dans Michel Bruneau (dir.), Les Grecs pontiques : diaspora, identité, territoires, CNRS, Paris, 1998, pp. 71-88. (infra : « La langue pontique... »

24. Publié chez Dr. Ludwig Reichert, Wiesbaden, 322 p.

25. Ibid. p. 373.

26. Gülden Aydın, «Pontus Müslümanları », Aktüel, 2 juillet 1992. En effet, la journaliste découvrait que dans ce village d'Iskenderli (Skandarando) les habitants communiquaient entre eux en grec, mais surtout il fallait passer par des interprètes pour s'entretenir avec certaines personnes. En réalité ce phénomène est beaucoup plus courant qu'on le pense surtout chez les personnes qui ne sont pas solarisées.

27. «Greek-Speaking Moslems of North-East Turkey : Prologomena to Study of the Ophitic SubDialect of Pontic », Byzantione and Modern Greek Studies, volume, 11, 1987, p. 115-137

28. Aspects pontiques, APR, Paris, 1997, XXVIII+791 p.

29. G. Drettas, La langue pontique..., p. 82.

30. Id.

31. Ce texte est rapporté par Vahit Tursun, dans son site internet : http://www.ocena.info. Je le remercie de m'avoir autorisé de reproduire ce texte et d'avoir corrigé ma traduction.

32. Ce second texte est un court extrait d'un long entretien que j'ai réalisé avec l'une de mes tantes habitant Istanbul en août 1989.

33. Je remercie Christina Alexopoulos et surtout mon collègue et ami Georges Galanès pour leur aide précieuse dans la transcription de ces textes en caractères grecs.

34. Peter Mackridge, « Greek-Speaking Moslems of North-East Turkey : Prologomena to Study of the Ophitic Sub-Dialect of Pontic ", Byzantione and Modern Greek Studies, volume, 11, 1987, p. 115-137

35. Ö. Asan, op. cit, p. 253-266.

36. Kurdret Emiroğlu, Trabzon-Maçka Etimoloji Sözlüğü (Dictionnaire ethymologique de TrabzonMaçka), Bülent Ofset, [Ankara], 1989.

37. Trabzon Il Yıllığı, 1973, p. 52-96.

38. Ö. Asan, op. cit, p. 136-137.

39. Gülden Aydın, op. cit., p. 58.

40. Pour l'ensemble des résultats électoraux officiels voir le site de la Haute Cour aux élections (Yüksek Seçim Kurulu) : http://www.ysk.gov.tr 


\section{RÉSUMÉS}

Une mise au point sur les travaux concernant la langue et la culture pontique qui ne demandent qu'à renaître en Turquie comme en Grèce.

Cet article est un premier essai socio-historique sur une langue et une culture, la langue pontique, qui ne demande qu'à renaître de ses cendres aussi bien en Turquie qu'en Grèce. Dans les deux pays en effet, les études scientifiques ont été «brouillées » par les nationalismes ambiants qui ont travesti ou dévalorisé un phénomène qui ne cadrait pas avec leurs interprétations $\mathrm{du}$ monde: l'existence d'une population musulmane de langue grecque. Cependant des travaux récents (et contestés en Turquie) permettent de sortir de cette situation, cet article fait le point sur l'état des travaux concernant l'historiographie, la langue, la toponymie et même l'onomastique et conclut en appelant à des études pluridisciplinaires sur ce potentiel historique, linguistique et sociologique en voie de disparition.

This article is an initial socio-historical investigation of a language and a culture, the Pontic language, which awaits rebirth from the ashes in Turkey as well as Greece. In both countries, scholarship has been 'scrambled' by the nationalisms that misrepresented or devaluated a phenomenon that did not tally with their interpretation of the world: the existence of a Greekspeaking Muslim population. While some recent studies (contested in Turkey) have pointed the way out of this dilemma, the present one takes stock of works on the historiography, the language, the toponomy and even the onomastics, and ultimately calls for multi-disciplinary studieson this historical, linguistic and sociological evidence, which is rapidly disappearing.

\section{INDEX}

motsclestr Karadeniz, Maçka, Of, Pontos, Samsun, Tonya, Trabzon, Türkiye

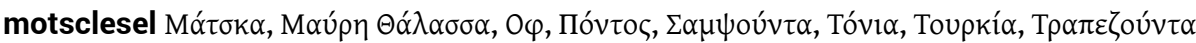

Mots-clés : Grande Idée, culture pontique, langue pontique, Millet, PKK, Pont/Pontique, Rum/

Roum

motsclesmk ГРЦИЈА, МАЛА АЗИЈА, ТУРЦИЈА, ЦРНОТО МОРЕ

Thèmes : Histoire, Linguistique

Index chronologique : dix-neuvième siècle, vingtième siècle

Index géographique : Asie Mineure, Grèce, mer Noire, Of, Samsun, Trébizonde, Turquie

Keywords : pontus linguage, pontus culture, Asia Minor, Greece, Turkey, Maçka, Black Sea, of, Pontus, Samsun, Tonya, Trabzon, nineteenth century, twentieth century, History 